\title{
O papel do Enfermeiro na prevenção do câncer de pele na Atenção Primária em
}

\section{Saúde}

\author{
The Nurse's role in skin cancer Prevention in Primary Health Care
}

El papel de la Enfermera en la prevención del cáncer de piel en la Atención Primaria de Salud

Vanessa de Oliveira Bezerra

ORCID: https://orcid.org/0000-0002-9776-0377 Instituto Brasileiro de Medicina e Reabilitação, Brasil E-mail: vans131417@gmail.com

Aline Maria Afonso Lino

ORCID: https://orcid.org/0000-0002-2049-3952 Instituto Brasileiro de Medicina e Reabilitação, Brasil

E-mail: loslinos28@gmail.com

Rafaela Lima de Oliveira

ORCID: https://orcid.org/0000-0002-0994-3246 Instituto Brasileiro de Medicina e Reabilitação, Brasil

E-mail: rafaela.limaoliveira@yahoo.com.br

Luciano Godinho Almuinha Ramos

ORCID: https://orcid.org/0000-0001-9958-3151 Instituto Brasileiro de Medicina e Reabilitação, Brasil E-mail: lucianogodinho@yahoo.com.br

\begin{abstract}
Resumo
Este estudo versa sobre o papel do enfermeiro na prevenção do câncer de pele na Atenção Primária em Saúde. Objetivase destacar a importância do profissional enfermeiro frente a prevenção do câncer de pele, na Atenção Primária. Para isso, foi utilizada a seguinte questão norteadora: Qual a importância do enfermeiro na prevenção do câncer de pele voltada para a Atenção Primaria em Saúde? Como critérios de inclusão foram utilizados artigos científicos entre os anos de 2015 à 2020, nas bases de dados, LILACS, SCIELO, BVS e BVS oncologia, em inglês, francês e espanhol. Como critérios de exclusão foram excluídos artigos de revisão fora do espaço temporal de pesquisa e que não abordavam a temática. Os resultados obtidos através dos 18 artigos científicos selecionados, trouxeram efetivação a elaboração de três categorias temáticas trazendo como principal foco o papel do enfermeiro na prevenção do câncer de pele na Atenção Primária. Conclui-se que a atuação do enfermeiro na atenção primária na conscientização da população sobre o câncer de pele e o estímulo às mudanças de comportamento são de fundamental importância para sua prevenção.
\end{abstract}

Palavras-chave: Papel do enfermeiro; Câncer de pele; Atenção primária.

\begin{abstract}
This study deals with the role of nurses in the prevention of skin cancer in Primary Health Care. The objective is to highlight the importance of the professional nurse regarding the prevention of skin cancer in Primary Care. For this, the following guiding question was used: What is the importance of nurses in the prevention of skin cancer focused on Primary Health Care? As inclusion criteria, scientific articles were used between the years 2015 to 2020 , in the databases, LILACS, SCIELO, BVS and BVS oncology in English, French and Spanish. As exclusion criteria, review articles outside the research time frame and that did not address the theme were excluded. The results obtained through the 18 selected scientific articles, brought into effect the elaboration of tree thematic categories, with the main focus being the role of nurses in the prevention of skin cancer in Primary Care. It is concluded that the role of nurses in primary care in raising awareness of the population about skin cancer and encouraging changes in behavior are of fundamental importance for its prevention.
\end{abstract}

Keywords: Nurse's role; Skin cancer; Primary attention.

\section{Resumen}

Este estudio trata sobre el papel del enfermero en la prevención del cáncer de piel en Atención Primaria de Salud y tiene como objetivo resaltar la importancia del profesional de enfermería en la prevención del cáncer de piel en Atención Primaria. Para ello, se utilizó la siguiente pregunta orientadora: ¿Cuál es la importancia del enfermero en la prevención del cáncer de piel con foco en Atención Primaria de Salud? Como criterio de inclusión se utilizaron artículos científicos entre los años 2015 a 2020, en las bases de datos LILACS, SCIELO, BVS y BVS oncología, en inglés, francés y español. Como criterios de exclusión, se excluyeron los artículos de revisión fuera del marco temporal de la investigación y que 
no abordaran el tema. Los resultados obtenidos a través de los 18 artículos científicos seleccionados propiciaron la elaboración de tres categorías temáticas con el foco principal en el papel del enfermero en la prevención del cáncer de piel en Atención Primaria. Se concluye que el papel del enfermero de atención primaria en la sensibilización de la población sobre el cáncer de piel y el fomento de cambios de comportamiento son de fundamental importancia para su prevención.

Palabras clave: Rol de la enfermera; Cáncer de piel; Atención primaria.

\section{Introdução}

O câncer de pele é o tipo de câncer mais comum no mundo e corresponde a cerca de $30 \%$ de todos os tumores malignos registrados no Brasil, o câncer de pele tem dois subtipos, o melanoma e o não melanoma.

De acordo com Brasil (2020), a exposição excessiva ao sol e sem o uso de filtro solar são fatores de risco para desenvolver o câncer de pele. Em um país de clima tropical como o Brasil é preciso ficar a alerta para os sintomas da doença e a importância do diagnóstico precoce. Por isso, estar informado sobre a doença é a melhor maneira de prevenção, diagnóstico e tratamento.

O câncer de pele do tipo melanoma representa apenas 3\% das neoplasias malignas do órgão, é o tipo mais grave devido à sua alta possibilidade de provocar metástase, ele tem origem nos melanócitos, células produtoras de melanina, substância que determina a cor da pele e é mais corriqueiro em adultos brancos. O melanoma pode aparecer em qualquer parte do corpo, na pele ou mucosas, na forma de manchas, pintas ou sinais, nos indivíduos de pele negra, ele é mais comum nas áreas claras, como palmas das mãos e plantas dos pés.

Segundo Brasil (2021), o câncer de pele não melanoma é o mais frequente no Brasil, apresenta altos percentuais de cura, se for detectado e tratado precocemente. De acordo com Brasil (2018), entre os tumores de pele este é o mais frequente e de menor mortalidade, porém, se não tratado adequadamente pode deixar mutilações bastante expressivas. Pessoas de pele clara, sensíveis à ação dos raios solares, com história pessoal ou familiar deste câncer ou com doenças cutâneas prévias são as mais atingidas, o câncer de pele não melanoma apresenta tumores de diferentes tipos, sendo os mais costumeiros o carcinoma basocelular e o carcinoma epidermoide.

Segundo Oliveira Junior, et al. (2019), dentre os principais fatores de risco para o desenvolvimento do câncer de pele temos o envelhecimento, exposição prolongada ao sol, possuir pele e olhos claros e muitos nevos (pintas) pelo corpo, câmaras de bronzeamento artificial, onde as mesmas foram reclassificadas pela Organização Mundial de Saúde (OMS) no mesmo patamar do cigarro e do sol, pois a prática de bronzeamento artificial antes dos 35 anos aumenta em 75\% as chances de um desenvolvimento de câncer de pele, as principais ações para o controle do câncer de pele concentram-se na prevenção e na detecção precoce, entre educador e educado com a finalidade não apenas de informar, mas principalmente de trocar experiências e reconhecimento que favoreçam a promoção de hábitos saudáveis de vida. (Biblioteca Virtual de Enfermagem, 2017)

A prevenção deve ser feita, de acordo com Oliveira, et al. (2021), principalmente por meio da foto proteção, sendo recomendada a utilização de chapéu e protetor solar diariamente, também se deve evitar a exposição à radiação artificial ultravioleta encontrada no processo de bronzeamento artificial. Segundo Purim, et al. (2020), deve-se focar na diminuição de fatores de risco relacionados ao ambiente e à ocupação dos indivíduos, como a exposição a compostos químicos, tais como agrotóxicos, carvão e outros, e exposição prolongada aos raios ultravioletas, nesse sentido, torna-se necessário que o enfermeiro esteja apto a reconhecer e a ensinar à população os principais sinais e sintomas desse tumor, a fim de possibilitar o reconhecimento dessas lesões, além de possibilitar a identificação dos casos suspeitos o mais precocemente possível.

\section{Metodologia}

Segundo Pereira, et al. (2018), uma das principais características do conhecimento científico é a sua estruturação, pois consiste num saber ordenado, o qual é construído a partir de um conjunto de ideias. Outra característica do conhecimento 
científico é ser verificável, isto é, determinada ideia deve ser verificada e comprovada sob a perspectiva da ciência para que possa fazer parte do conhecimento científico.

Segundo Souza, et al. (2010), a revisão integrativa é um método que proporciona a síntese de conhecimento e a incorporação da aplicabilidade de resultados de estudos significativos na prática.

Para alcançar o objetivo proposto, foi utilizado uma revisão integrativa da literatura e para a seleção dos artigos foram acessadas as seguintes bases de dados: Biblioteca virtual em saúde (BVS), BVS oncologia, Literatura Latino- Americana e do Caribe em Ciências da Saúde (LILACS), Scientific Electronic Library Online (SCIELO) e Instituto Nacional do Câncer Jose Alencar Gomes da Silva (INCA), onde todo o processo de desenvolvimento da pesquisa e seleção dos artigos foi conduzido por três pesquisadores independentes. No processo de montagem, foram encontrados 93 artigos, sendo 32 na BVS, 41 no LILACS e 20 no SCIELO.

Foi escolhida a seguinte questão norteadora: Qual a importância do enfermeiro na prevenção do câncer de pele, voltada para a Atenção Primaria em Saúde? Com a busca dos estudos selecionados conforme os critérios de inclusão, foi utilizado artigos científicos entre os anos de 2015 a 2021, em inglês, espanhol e francês, artigos em sua versão na íntegra, artigos originais que englobassem os objetivos propostos e abordassem o tema: O papel do enfermeiro na prevenção do câncer de pele na atenção primária em saúde, totalizando 32 artigos.

Conforme os critérios de exclusão, os artigos que não apresentavam a temática, artigos de revisão, artigos que não se relacionavam aos objetivos desta pesquisa, dissertações ou teses, foram descartados. Dessa forma foram excluídos um total de 13 artigos. Sendo que, após a leitura, foram selecionados 18 artigos para a elaboração final do estudo pelos bancos de dados.

A figura a seguir, apresenta como foi realizada a seleção dos artigos que compuseram a amostra final da revisão.

Figura 1. Fluxograma de busca e seleção dos 18 artigos. Rio de janeiro, RJ, Brasil, 2021.

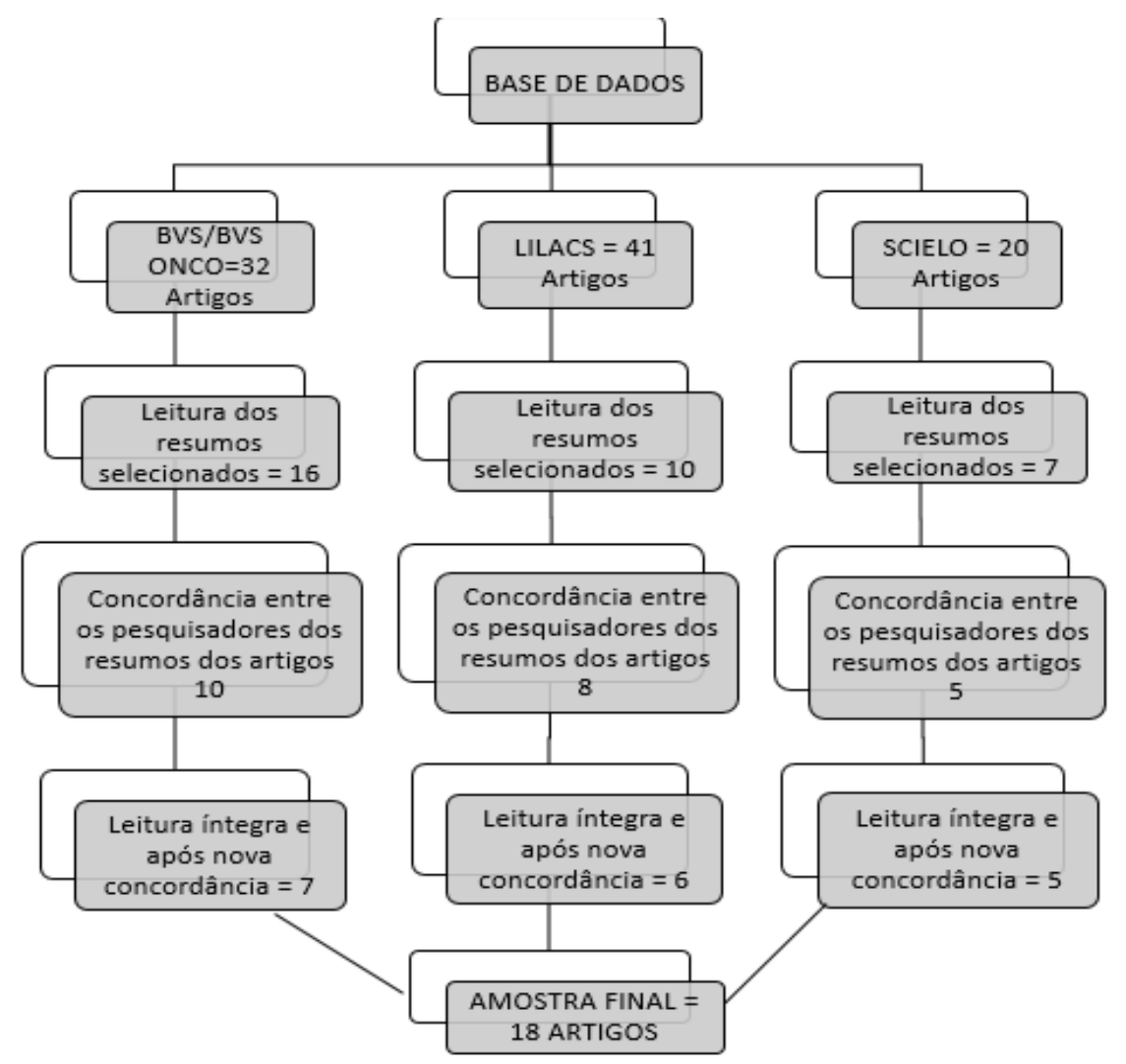

Fonte: Dados da pesquisa. 
Analisando o Fluxograma 1, podemos observar os artigos que levaram a elaboração desta revisão, juntamente com a ordem de busca dos mesmos, verificando, banco de dados, seleção, exclusão e escolha para a obtenção da amostra final.

\section{Resultados e Discussão}

Analisando o Quadro 1, podemos observar o esqueleto de organização e estrutura de seleção dos artigos, compondo o desenvolvimento desta revisão integrativa.

A busca dos artigos científicos foi realizada entre março e abril de 2021, e para a apresentação das etapas de seleção dos artigos de acordo com as informações adquiridas, foi elaborado um quadro como metodologia com os principais dados dos artigos científicos escolhidos.

Dos 18 artigos selecionados no campo deste estudo, todos tiveram publicação entre 2015 e 2020 , sendo em maior quantidade as apresentações dos artigos de 2017 e 2018.

Quadro 1. Tabela expositiva dos artigos pesquisados neste estudo. Rio de Janeiro, RJ, Brasil, 2021.

\begin{tabular}{|c|c|c|}
\hline AUTOR/ANO & TÍTULO & OBJETIVOS \\
\hline PURIM, K.S.M., et al. (2020) & $\begin{array}{l}\text { Características do melanoma em } \\
\text { idosos. }\end{array}$ & Avaliar características do melanoma em idosos. \\
\hline CRUZ, G. T. A., et al. (2020) & $\begin{array}{l}\text { Fatores associados ao uso do } \\
\text { protetor solar como medida de } \\
\text { prevenção aos danos causados } \\
\text { pela exposição solar. }\end{array}$ & $\begin{array}{l}\text { Investigar os fatores associados ao uso do } \\
\text { fotoprotetor solar como na região metropolitana } \\
\text { de Belém. }\end{array}$ \\
\hline $\begin{array}{l}\text { OLIVEIRA Junior, E.F.S., et al. } \\
\text { (2019) }\end{array}$ & $\begin{array}{l}\text { Educação em saúde do paciente } \\
\text { com diagnóstico de câncer de pele. }\end{array}$ & $\begin{array}{l}\text { Realizar uma análise sobre a educação em saúde } \\
\text { oferecida na atenção primaria a pacientes } \\
\text { diagnosticados com câncer de pele. }\end{array}$ \\
\hline CARMO, R.A.L.O., et al. (2019) & $\begin{array}{l}\text { Cuidar em Oncologia: Desafios e } \\
\text { Superações } \quad \text { Cotidianas } \\
\text { Vivenciados por Enfermeiros. }\end{array}$ & $\begin{array}{l}\text { Compreender a perspectiva de enfermeiros acerca } \\
\text { do processo de enfrentamento dos desafios } \\
\text { vivenciados no cuidado à pessoa com câncer. }\end{array}$ \\
\hline CAUDURO, F.P., et al. (2018) & $\begin{array}{l}\text { Atuação dos enfermeiros no } \\
\text { cuidado das lesões de pele. }\end{array}$ & $\begin{array}{l}\text { Conhecer a atuação dos enfermeiros no cuidado } \\
\text { aos pacientes com lesões de pele. }\end{array}$ \\
\hline FOLONI, A.R., et al. (2018) & $\begin{array}{l}\text { Analise de casos de câncer de pele } \\
\text { em um hospital do interior } \\
\text { paulista. }\end{array}$ & $\begin{array}{l}\text { Avaliar as estatísticas dos casos de câncer de pele } \\
\text { em um hospital escola, a fim de contribuir na } \\
\text { programação de ações voltadas à prevenção e } \\
\text { controle da doença. }\end{array}$ \\
\hline PIRES, C.A.A., et al. (2018) & $\begin{array}{l}\text { Câncer de pele: caracterização do } \\
\text { perfil e avaliação da proteção } \\
\text { solar dos pacientes atendidos em } \\
\text { serviço universitário. }\end{array}$ & $\begin{array}{l}\text { Descrever os casos de câncer de pele, bem como } \\
\text { o hábito de proteção solar na prevenção de lesões } \\
\text { causadas por radiação ultravioleta. }\end{array}$ \\
\hline SCHMIDT, F.M.Q., et al. (2018) & $\begin{array}{l}\text { Conhecimento da equipe de } \\
\text { enfermagem sobre cuidados com } \\
\text { pacientes com feridas neoplásicas. }\end{array}$ & $\begin{array}{l}\text { Avaliar o conhecimento da equipe de enfermagem } \\
\text { de um hospital oncológico sobre o cuidado de } \\
\text { pacientes com Feridas Neoplásicas Malignas } \\
\text { (FNM) e analisar fatores sociodemográficos e } \\
\text { educacionais associados. }\end{array}$ \\
\hline BELTRÃO, T.A., et al. (2018) & $\begin{array}{l}\text { Acompanhamento de pessoas com } \\
\text { câncer por enfermeiros da atenção } \\
\text { primária }\end{array}$ & $\begin{array}{l}\text { Compreender o processo de acompanhamento de } \\
\text { pessoas com câncer por enfermeiros da atenção } \\
\text { primária. }\end{array}$ \\
\hline SANTOS, R.O.M. D., (2017) & $\begin{array}{l}\text { Atuação do enfermeiro na } \\
\text { detecção precoce do câncer de } \\
\text { pele. }\end{array}$ & $\begin{array}{l}\text { Analisar a produção científica na } \\
\text { área da enfermagem em relação à detecção precoce } \\
\text { do câncer de pele. }\end{array}$ \\
\hline
\end{tabular}




\begin{tabular}{|c|c|c|}
\hline $\begin{array}{l}\text { ESPOSITO, A.C.C., et al. } \\
(2017)\end{array}$ & $\begin{array}{l}\text { Fatores que levam a negligencia } \\
\text { quanto aos cânceres da pele não } \\
\text { melanoma }\end{array}$ & $\begin{array}{l}\text { Pontuar através de estudos de caso, os fatores que } \\
\text { levam ao negligenciamento do CPNM. }\end{array}$ \\
\hline SOUZA, G.R.M., et al. (2017) & $\begin{array}{l}\text { Atuação dos enfermeiros da } \\
\text { estratégia saúde da família na } \\
\text { atenção oncológica. }\end{array}$ & $\begin{array}{l}\text { Identificar a qualificação e conhecer a atuação dos } \\
\text { enfermeiros da Estratégia Saúde da Família na } \\
\text { atenção oncológica. }\end{array}$ \\
\hline WAKIUCHI, J. et al (2016) & $\begin{array}{l}\text { Atenção a pacientes oncológicos } \\
\text { na Estratégia Saúde da Família: } \\
\text { olhar do usuário }\end{array}$ & $\begin{array}{l}\text { Compreender as experiências de pacientes com } \\
\text { câncer referentes aos cuidados recebidos e a } \\
\text { relação com os profissionais da Estratégia Saúde } \\
\text { da Família. }\end{array}$ \\
\hline BARATTO, F. et al. (2016) & $\begin{array}{l}\text { Humanização da assistência na } \\
\text { atenção básica às pessoas com } \\
\text { neoplasia: percepção de } \\
\text { profissionais de enfermagem. }\end{array}$ & $\begin{array}{l}\text { Conhecer a percepção de profissionais de } \\
\text { enfermagem atuantes na atenção básica acerca da } \\
\text { humanização da assistência às pessoas com } \\
\text { neoplasias. }\end{array}$ \\
\hline WAKIUCHI, J. et al. (2016) & $\begin{array}{l}\text { Atuação da estratégia saúde da } \\
\text { família na perspectiva de usuários } \\
\text { com câncer. }\end{array}$ & $\begin{array}{l}\text { Apreender como usuários com câncer percebem a } \\
\text { atuação das equipes da Estratégia Saúde da } \\
\text { Família. }\end{array}$ \\
\hline MOREIRA, A.P.A., et al. (2015) & $\begin{array}{l}\text { Câncer de pele não melanoma e } \\
\text { risco ocupacional de } \\
\text { trabalhadores ao ar livre. }\end{array}$ & $\begin{array}{l}\text { Analisar a relação do câncer de pele não melanoma } \\
\text { e os riscos ocupacionais dos trabalhadores } \\
\text { expostos ao ar livre. }\end{array}$ \\
\hline $\begin{array}{l}\text { MATHEUS, L.G.M., et al. } \\
(2015)\end{array}$ & $\begin{array}{l}\text { Aspectos epidemiológicos do } \\
\text { melanoma cutâneo. }\end{array}$ & $\begin{array}{l}\text { Analisar aspectos epidemiológicos do melanoma } \\
\text { cutâneo. }\end{array}$ \\
\hline $\begin{array}{l}\text { CEZAR-VAZ, M.R., et al. } \\
(2015)\end{array}$ & $\begin{array}{l}\text { Câncer de pele em trabalhadores } \\
\text { rurais: conhecimento } \quad e \\
\text { intervenção de enfermagem. }\end{array}$ & $\begin{array}{l}\text { Identificar a exposição de trabalhadores rurais à } \\
\text { radiação ultravioleta solar e pesticidas; identificar } \\
\text { casos anteriores de câncer de pele; implementar } \\
\text { clínica e ações comunicativas de enfermagem entre } \\
\text { trabalhadores rurais com diagnóstico prévio de } \\
\text { câncer de pele. }\end{array}$ \\
\hline
\end{tabular}

Fonte: Dados da pesquisa.

Os resultados obtidos através dos 18 artigos científicos selecionados, trouxeram efetivação a elaboração de três categorias temáticas: $\mathrm{O}$ papel do enfermeiro frente ao câncer de pele na atenção primária, Melanoma: O enfermeiro em sua identificação e A humanização do atendimento na percepção do enfermeiro na atenção primária.

\section{Categoria 1 - O papel do enfermeiro frente ao câncer de pele na Atenção Primária}

Ao analisar os 18 artigos selecionados foi possível evidenciar que compete à Atenção Primária, as ações de diagnóstico precoce. Nesse sentido, torna-se necessário que o enfermeiro transmita à população o conhecimento necessário para identificação dos sinais e sintomas de Câncer de pele, possibilitando o reconhecimento dessas lesões e a identificação dos casos suspeitos o mais precocemente possível.

O profissional de enfermagem é essencial na detecção precoce do câncer de pele segundo Purim, et al. (2020), uma vez que está inserido diretamente na área do cuidado, atuando diretamente na prevenção e na assistência dos usuários nos diversos pontos da atenção primária. (Baratto, et al. 2016.)

O enfermeiro na Atenção Primaria em Saúde (APS), vêm conquistando espaço social e reconhecimento junto aos integrantes da equipe de saúde e dos usuários que vivenciam com ele o atendimento clínico e identificam nele a referência para o seu cuidado, o que traz muita satisfação e dá sentido ao trabalho.

Observa-se que o profissional de enfermagem é um dos primeiros a ter contato com os pacientes que procuram assistência de uma equipe de cuidados específicos segundo os estudos de Schmidt, F. M.Q., et al. (2020). Esse profissional de acordo com Cauduro, et al. (2018), ocupa uma posição privilegiada para realizar a avaliação visual da pele e obter a história 
clínica dos pacientes dentro das unidades de pronto atendimento, segundo Cruz, et al. (2020) este profissional possui um campo fértil de atuação para desenvolver ações de educação em prevenção e detecção precoce do câncer de pele, propício à realização de ações que, junto à equipe de atenção primaria na RAS, busquem controlar a incidência e a mortalidade do câncer no país.

O autoconhecimento do profissional de saúde é importante para que se estabeleça um relacionamento interpessoal adequado ao processo de cuidar, desenvolvendo consciência de suas limitações, fragilidades e potencialidades. (Matheus, et al. 2015.)

O conhecimento abrangente e profundo dos fatores relacionados e dos problemas que afetam a saúde da população atendida pela instituição, como sua condição socioeconômica, necessidades e carências, crenças e valores culturais, entre outros aspectos, contribui para que os profissionais se tornem mais comprometidos com a população atendida e com a busca de resultados concretos e coerentes com a realidade de vida das pessoas sob seus cuidados.

Segundo Macena, et al. (2020), na oncologia, os cuidados técnicos são de extrema importância para o prognóstico da pessoa, entretanto, apenas essa modalidade de cuidado não supre as necessidades psicológicas, emocionais, sociais, culturais e as crenças inerentes a cada ser, assim, evidencia-se que o conhecimento técnico do enfermeiro e a humanização precisam estar interligados para prover melhor qualidade de vida ao usuário. (Araújo, et al. 2016)

De acordo com Souza, et al. (2017), a maneira como as relações entre a enfermagem e os usuários dos serviços de saúde ocorrem é fundamental para a produção do cuidado, quando esta relação é realizada no trabalho vivo em ato; segundo Wakiuchi, et al. (2016) e Bica, et al. (2020), a enfermagem conta com altos graus de liberdade, podendo exercer melhor a criatividade necessária ao cuidado em saúde e fortalecer os aspectos relacionais do seu trabalho, promovendo abertura para o encontro da produção do cuidado que atenda às reais expectativas dos usuários na unidade de atendimento.

Sendo uma profissão fundamental no sistema de saúde, a enfermagem se destaca e diferencia pelo desenvolvimento de práticas interativas e integradoras de cuidado, às quais vêm adquirindo uma repercussão cada vez maior, tanto na educação e promoção da saúde, quanto no fomento de políticas voltadas para o bem-estar social das famílias e comunidades.

A Atenção Primária é responsável, dentre outras demandas, por ações direcionadas ao usuário com câncer e aos seus familiares de acordo com Gomide, et al. (2018), devendo se fazer presente em todas as fases da doença, o que demonstra que o enfermeiro tem um papel importante no atendimento, tanto durante a consulta de enfermagem, diagnóstico, quanto ao tratamento do câncer nos pacientes. (Wakiuchi, et al. 2016.)

O plano de cuidados de enfermagem ao usuário da Atenção Primária na prevenção do câncer de pele tem como prioridade o acolhimento de acordo com Ribeiro, et al. (2016), e segundo Carmo, et al. (2019), com a postura ética, que integre o usuário como protagonista em seu processo terapêutico, considerando sua cultura, seus saberes e sua capacidade de avaliar riscos, respeitando seus medos e fragilidades o planejamento da enfermagem torna-se efetivo. Esposito, et al. (2017), relata nesta perspectiva que a equipe de enfermagem é fundamental e a primordial referência na atenção diária, responsável e gestora desse processo.

O acolhimento deve ser entendido como diretriz ética, estética e política constitutiva dos modos de se produzir saúde, além de ferramenta tecnológica de intervenção na qualificação de escuta, construção de vínculo, garantia do acesso com responsabilização e resolutividade nos serviços. (Cezar-Vaz, et al. 2015)

Para que as necessidades básicas apresentadas pelos usuários sejam sanadas, é necessário que haja interação entre o profissional e o usuário, sendo assim, podemos dizer que a criação do vínculo do enfermeiro para com o cliente favorece a construção de confiança e compromisso entre os usuários e a enfermagem, facilitando o acesso ao serviço e as relações interpessoais. 
Segundo Moreira, al. (2015), atribui-se ao enfermeiro o cuidado ao paciente, esse profissional pode realizar consulta de enfermagem, prescrever e executar curativos, coordenar e supervisionar a equipe de enfermagem na prevenção e cuidados referentes a neoplasias de pele, dentre outras atribuições específicas. (Pires, et al. 2018).

Na Atenção Primária, o enfermeiro é responsável pelo planejamento, organização, execução e avaliação da assistência dispensada aos pacientes visando a um cuidado seguro e de qualidade, unindo e ampliando as estratégias de sistematização do cuidado da pele, avaliação e classificação das lesões, tratamento adequado e recuperação. (Silva, et al.2017).

A equipe da APS conta com ativa participação do enfermeiro, que realiza, entre outras ações, consulta de enfermagem e atividades de educação. (Cavalcante, et al. 2015)

O enfermeiro tem autonomia para desenvolver atividades variadas, isso envolve o cargo a que ele está inserido, mas em todos eles ele desenvolve atividades do aperfeiçoamento do pessoal e manutenção das condições para a prestação de um atendimento com excelência.

A importância de conhecer as práticas de atenção à saúde e a maneira como se efetivam no cotidiano do trabalho para a produção do cuidado tanto para a área da gestão quanto da organização dos serviços de saúde, são de relevância para a configuração da integralidade, não somente como princípio do SUS, mas também como boas práticas de saúde para a melhoria do cuidado. (Valentim, et al. 2020).

De acordo com Foloni, et al. (2018) as Unidades de Atenção Primária à Saúde, são consideradas porta de entrada do usuário no sistema de saúde, espaço em que o enfermeiro é importante integrante da equipe multiprofissional da Estratégia Saúde da Família, o enfermeiro que está inserido na atenção primária tem o papel de gerente e provedor da assistência à saúde. (Lopes, et al. 2020).

A responsabilidade do enfermeiro é de contribuir com a compreensão da realidade em todas as suas dimensões, é parte das atribuições do enfermeiro, realizar assistência integral aos usuários que procuram a unidade de saúde, neste caso, a realização da consulta de enfermagem com atenção voltada a identificação, prevenção, educação em saúde e tratamento das neoplasias de pele. (Beltrão, et al. 2020).

\section{Categoria 2 - Melanoma: $O$ papel do enfermeiro em sua identificação}

Os diferentes tipos de câncer de pele fundam-se a partir das camadas celulares. Estes se dividem Câncer de pele do tipo Não Melanoma (CPNM) e o câncer de pele tipo melanoma (CPM).

O tipo não melanoma se subdivide em carcinoma basocelular representando o tipo de câncer de pele mais comum, apresenta-se principalmente como uma lesão (ferida ou nódulo) com as bordas róseas, translucidas ou peroladas, que não cicatriza, podendo ulcerar e sangrar, já o epidermoide, em geral surge como uma mancha vermelha que descama e sangra formando uma ferida que muitas vezes aparece sobre uma cicatriz, principalmente aquelas recorrentes a queimaduras. (Brasil, 2018).

Então consideramos importante que um conhecimento ou um plano preventivo do câncer na pele seja de suma importância para toda a população, como também aos profissionais de saúde da região, pois os indicadores de saúde demostram um aumento na população pelos fatores genéticos e ambientais, desta forma uma correta educação em saúde para população auxilia ao diagnóstico precoce do câncer de pele, pois 78\% dos canceres de pele, originam-se das células da camada basal da epiderme, onde raramente ocorre metástase, diferentemente dos carcinomas de células escamosas, que equivalem aproximadamente a $20 \%$ de todos os cânceres de pele que possuem tendências para a metástase.

O câncer de pele do tipo melanoma, conhecido também como melanoma maligno é o mais perigoso, surge como uma pinta escura que se deforma ao longo do tempo podendo ser fatal se não for diagnosticado precocemente, pois o mesmo pode se desenvolver rapidamente atingindo outros órgãos. (Santos, 2017) 
A presença de assimetria (A), bordas irregulares e mal definidos (B), alterações de cor (C), diâmetro maior que $6 \mathrm{~mm}$ (D) e evolução/ evolução recente da lesão (E) constituem o $\mathrm{ABCDE}$ dos diagnósticos do melanoma que deve ser confirmado por análise histopatológica da lesão.

O melanoma é uma doença perigosa e as pessoas precisam saber identificar quando alguma pinta mudou na sua pele. A pinta suspeita nem sempre vai se transformar em um melanoma. Porém, quanto antes iniciar-se um diagnóstico e um tratamento, mais tranquilo será o processo, então cabe a enfermagem saber identificar as lesões, e um conhecimento relacionado ao $\mathrm{ABCDE}$ é de suma importância.

O melanoma é classificado em extensivo superficial, nodular, acrolentiginoso e lentigo maligno melanoma, podendo ocorrer outras apresentações e diferente tipo entre as faixas etárias. Com a senescência o sistema imune diminui a resposta contra neoplasias, favorecendo alta mortalidade nessa faixa etária, além disso, idosos apresentam maior índice de Breslow e metástase ao diagnostico inicial. (Oliveira, et al. 2021)

O Instituto Nacional do Câncer (INCA) afirma que o câncer de pele não melanoma é o mais incidente no Brasil em ambos os sexos, mesmo considerando que estes índices podem estar subestimados pelo fato de que muitas lesões suspeitas são retiradas sem diagnóstico. Este tipo de câncer apresenta ainda a propriedade de possuir baixa letalidade, sendo esse um tumor relativamente fácil de diagnóstico precocemente, porque está exposto e facilmente visível. Porém em alguns casos podem levar a deformidade física e ulcerações graves, consequentemente, onerando os serviços de saúde. (Foloni, et al. 2018)

\section{Categoria 3 - A Humanização do atendimento na percepção do enfermeiro na atenção primária.}

A temática humanização tem sido abordada com maior ênfase a partir da implementação pelo Ministério da Saúde em 2003, O Humaniza SUS, essa política tem como princípios norteadores a valorização da dimensão subjetiva e social em todas as práticas de atenção e gestão no Sistema Único de Saúde. (Brasil, 2015)

Como qualquer outro trabalhador da saúde, o enfermeiro precisa estar ciente e sensibilizado quanto à questão da humanização, saber reconhecer o ser humano na sua integridade e singularidade e ter consciência do seu papel frente aqueles que o procuram acometidos por alguma enfermidade, aceitando suas reações psíquicas e a própria atitude frente à doença.

Dentre as inúmeras doenças que acometem a população encontra-se o câncer, que, nas últimas décadas, ganhou maior dimensão, convertendo-se em problemas da saúde pública mundial. (Baratto, et al. 2016).

Os cuidados prestados pelo enfermeiro são realizados de diferentes formas, no atendimento direto tanto ao paciente quanto a família. (Macena, et al. 2020)

Sendo assim, o enfermeiro traz ao familiar a consciência da necessidade de apoio que essa deve dispensar ao doente com câncer uma vez que o enfrentamento poderá se tornar mais seguro e tranquilo, conduzindo essa pessoa a um tratamento e cuidado com algum conforto ao longo de sua caminhada pós-diagnóstico.

Segundo Foloni, et al. (2018), no campo da atenção primária à saúde, muitas práticas tem sido desenvolvidas por meio das Unidades Básicas de Saúde da família, sendo esta uma proposta de reorientação do modelo assistencial de saúde, a partir da Atenção Primária.

A enfermagem deve atuar junto aos usuários e seus familiares no sentido de apoiar os sentimentos, medos e ansiedade decorrente do adoecimento, realizando planejamento e traçando metas que venham minimizar as consequências a serem enfrentadas pela pessoa com câncer e sua família, promovendo a qualidade de vida e continuidade do cuidado. Desta forma, há a compreensão das estratégias utilizadas pelos enfermeiros da equipe da saúde da família no acompanhamento de pessoas com câncer, bem como das dificuldades para a implementação desse acompanhamento. 
De acordo com Beltrão, et al. (2019), o acompanhamento dos pacientes com câncer pela equipe tem o papel não só de estabelecer a construção e o fortalecimento do vínculo entre o usuário, família e unidade de saúde, como também permite que a equipe possa prestar uma assistência de qualidade priorizando a necessidade do indivíduo.

\section{Conclusão}

Os 18 artigos científicos selecionados destacaram que, entre as estratégias utilizadas pelo enfermeiro à atuação do cuidado da pele, a importância de manter-se atualizado estimulando o raciocínio clínico e buscando a melhor terapêutica como investir em educação permanente pode permitir a troca de experiências vivenciadas institucionalmente no cuidado da pele.

É evidente que o acolhimento é como uma ação que por meio de diálogos responsabiliza o usuário no processo da produção da saúde, ressalta-se que o conhecimento básico sobre a doença ajuda em suas possíveis prevenções. Neste caso, é de suma importância o apoio do enfermeiro para que possam fornecer orientações de saúde pertinentes às carências de cada indivíduo de acordo com seus conhecimentos básicos.

No cenário da prevenção do câncer de pele, a atuação do enfermeiro na atenção primária revelou-se de importância fundamental, como as ações educativas diversas junto à equipe de saúde e comunidade, como também ações preventivas ao câncer de pele.

Constatou-se que os fatores relacionados ao uso do protetor solar como forma preventiva aos danos gerados pela exposição solar, e o grau de informação do indivíduo para a realização do autocuidado juntamente à questão da renda do paciente, é um aspecto que pode influenciar no cuidado com a pele, uma vez que as pessoas favorecidas financeiramente têm acesso a melhores tipos de prevenção do que as menos favorecidas.

A prevenção representa uma estratégia promissora para enfrentar os múltiplos problemas de saúde que a população enfrenta, relacionando-se com os saberes técnicos e populares, mobilizando os recursos comunitários, públicos e privados, buscando sempre uma construção de propostas com o intuito de proporcionar um conhecimento mais adequado sobre o câncer de pele.

A conscientização da população sobre o câncer e o estímulo às mudanças de comportamento é de fundamental importância para sua prevenção, e neste processo o papel educativo da enfermagem na atenção primária merece destaque.

Tendo em vista os resultados obtidos neste trabalho, são feitas as seguintes sugestões para futuras pesquisas: o reconhecimento dos sinais e sintomas indicativos de câncer de pele, assim como o fácil acesso ao serviço de saúde especializado pelo enfermeiro, um acolhimento mais qualificado, onde deve ser feita, ainda, uma abordagem mais direta e clara em relação a prevenção a exposição aos raios UV, com o uso de protetores, como também a não exposição ao sol em horários específicos, estimulando, orientando e conscientizando a população sobre a prevenção deste tipo de câncer.

\section{Referências}

Araújo, M. P. S., et al. (2016). Relacionamento interpessoal da equipe de enfermagem: fragilidades e fortalezas. Rev enferm UERJ, 24(5):e7657. http://dx.doi.org/10.12957/reuerj.2016.7657

Baratto, F., et al. (2016). Humanização da assistência na atenção básica às pessoas com neoplasia: percepção de profissionais de enfermagem. Rev enferm UFPE on line, 10(2):615-22. https://periodicos.ufpe.br/revistas/revistaenfermagem/arti cle/view/10997/12354

Beltrão, T. A., et al. (2019). Acompanhamento de pessoas com câncer por enfermeiros da atenção primária. Revista cubana de enfermeira volumen 35, Número 4. http://www.revenfermeria.sld.cu/index.php/enf/article/view/3011/497

Biblioteca virtual de enfermagem, (2017). Fundação do Câncer Dá Dicas de Prevenção ao Câncer de Pele. http://biblioteca.cofen.gov.br/fundacao-do-cancerda-dicas-de-prevencao-ao-cancer-de-pele/

Bica, M. C., et al. (2020). Gerenciamento do cuidado em estratégias saúde da família na percepção de enfermeiros. Rev. Enferm. UFSM. 10, 1-18. https://periodicos.ufsm.br/reufsm/article/view/42518/html 
Brasil. (2015). Ministério da saúde. Política nacional de humanização- HumanizaSUS. https://www.gov.br/saude/pt-br/acesso-a-informacao/acoes-eprogramas/humanizasus\#: :text=A\%20Pol\%C3\%ADtica\%20Nacional\%20de $\% 20$ Humaniza\%C3\%A7\%

C3\%A3o,entre\%20gestores\%2C\%20trabalhadores\%20e\%20usu\%C3\%A1rios.

Brasil. (2018). Ministério da Saúde. Instituto Nacional de Câncer (INCA). Tipos de câncer: Câncer de pele não melanoma - versão para Profissionais de Saúde. https://www.inca.gov.br/tipos-de-cancer/cancer-de-pele-nao-melanoma/profissional-de-saude

Brasil. (2020). Ministério da Saúde. Câncer de pele: saiba como prevenir, diagnosticar e tratar- Saúde e vigilância sanitária. https://www.gov.br/saude/ptbr/assuntos/noticias/cancer-de-pele-saiba-como-prevenir-diagnosticar-e-tratar

Brasil. (2021). Ministério da Saúde. Instituto Nacional de Câncer (INCA). Tipos de câncer: Câncer de pele melanoma - versão para Profissionais de Saúde. https://www.inca.gov.br/tipos-de-cancer/cancer-de-pele-melanoma/profissional-de-saude

Carmo, R. A. L. O., et al. (2019). Cuidar em Oncologia: Desafios e Superações Cotidianas Vivenciados por Enfermeiros. Rev. bras. cancerol, 65(3)19/09/2019. https://www.inca.gov.br/tipos-de-cancer/cancer-de-pele-nao-melanoma/profissional-de-saude

Cauduro, F. P., et al. (2018). Atuação dos enfermeiros no cuidado das lesões de pele. Rev. enferm. UFPE on line; 12(10): 2628-2634. https://pesquisa.bvsalud.org/portal/resource/pt/biblio-996492

Cavalcante, A. K. C. B., et al. (2015). Cuidado seguro ao paciente: contribuições da enfermagem. Revista cubana de enfermeira volumen 31, Número 4. http://www.revenfermeria.sld.cu/index.php/enf/article/view/907/141

Cezar-Vaz, M. R., et al. (2015). Câncer de pele em trabalhadores rurais: conhecimento e intervenção de enfermagem. Rev Esc Enferm USP. 49(4):564-571. https://www.scielo.br/pdf/reeusp/v49n4/pt_0080-6234-reeusp-49-04-0564.pdf

Cruz, G. T. A., et al. (2020). Fatores associados ao uso do protetor solar como medida de prevenção aos danos causados pela exposição solar. Braz. J. of Develop. 6, 99546-99563. https://www.brazilianjournals.com/index.php/B RJD/article/view/ 21841/17430

Esposito, A. C. C., et al. (2017). Fatores que levam à negligência quanto aos cânceres da pele não melanoma. Diagn. tratamento ; 22(2): 63-66, https://pesquisa.bvsalud.org/portal/resource/pt/biblio-833682

Foloni, A. R., et al. (2018). Analise de casos de câncer de pele em um hospital do interior paulista. CuidArte, Enferm; 12(2): 175-180, https://pesquisa.bvsalud.org/portal/resource/pt/biblio-1005520

Gomide, M. F. S., et al. (2018). A satisfação do usuário com a atenção primária à saúde: uma análise do acesso e acolhimento. Artigos. Interface 22 (65). https://scielosp.org/article/icse/2018.v22n65/387-398/pt/

Lopes, O. C. A., et al. (2020). Competências dos enfermeiros na estratégia Saúde da Família. Esc Anna Nery 24(2): e20190145. https://www.researchgate.net/publication/339416912_Competencias_dos_enfermeiros_na_estrategia_Saude_da_Familia

Macena, A.A., et al. (2020). Aspectos emocionais da equipe de enfermagem frente aos cuidados em oncologia. Anais do congresso de geriatria e gerontologia do UNIFACIG, capa http://pensaracademico.unifacig.edu.br/index.php/congressogeriatri a/arti cle/view/2619

Matheus, L. G. M., et al. (2015). Aspectos epidemiológicos do melanoma cutâneo. Revista Ciência e Estudos Acadêmicos de Medicina - Número 3. Universidade do Estado de Mato Grosso - UNEMAT (Cáceres). (p. 10-24). https://periodicos.unemat.br/index.php/revistamedicina/article/view/625/543

Moreira, A. P. A., et al. (2015). Câncer de pele não melanoma e risco ocupacional de trabalhadores ao ar livre. J Nurs UFPE on line, 9(12):1310-9, https://periodicos.ufpe.br/revistas/revistaenfermagem/article/view/10839/12043

Oliveira, F. M. A., et al. (2021). Uso de medidas preventivas para câncer de pele por mototaxistas. Rev. Pesqui. (Univ. Fed. Estado Rio J., Online; $13: 282-287$. http://seer.unirio.br/index.php/cuidadofundamental/article/view/8526/9470

Oliveira Junior, E. F. S., et al. (2019). Educação em saúde do paciente com diagnóstico de câncer de pele. Nursing (Säo Paulo) ; 22(251): 2898-2903. https://pesquisa.bvsalud.org/portal/resource/pt/biblio-998735

Pereira A. S., et al. (2018). Metodologia da pesquisa científica. UFSM. https://repositorio.ufsm.br/bitstream/handle/1/158 24/Lic_Computa cao_MetodologiaPesquisa-Cientifica.pdf?sequence $=1$

Pires, C. A. A., et al. (2018). Câncer de pele: caracterização do perfil e avaliação da proteção solar dos pacientes atendidos em serviço universitário. J. Health Biol Sci. 6(1):54-59 https://periodicos.unichristus.edu.br/jhbs/article/view/1433/551

Purim, K. S. M., et al. (2020). Características do melanoma em idosos. Rev Col Bras Cir 47:e20202441. 10.1590/0100-6991e-20202441

Ribeiro, J. P., et al. (2016). Assistência de enfermagem ao paciente oncológico hospitalizado: diagnósticos e intervenções relacionadas às necessidades psicossociais e psicoespirituais. res.: fundam. care. http://www.seer.unirio.br/index.php/cuidad ofundamental/article/view/4016/pdf_1

Santos, R. O. M. D, (2017). Atuação do enfermeiro na detecção precoce do câncer de pele. Rev. baiana saúde pública ; 41(1): https://doi.org/10.22278/23182660.2017.v41.n1.a2331.

Schmidt, F. M. Q., et al. (2020). Conhecimento da equipe de enfermagem sobre cuidados com pacientes com feridas neoplásicas. Rev. Bras. Enferm. 73. https://www.scielo.br/scielo.php?pid=S003471672020000100154\&script=sci_arttext\&tlng=pt

Silva, S. S., et al. (2017). Enfermeira como protagonista do gerenciamento do cuidado na estratégia saúde da família: diferentes olhares analisadores. Texto Contexto Enferm, 26(3):e1090016. https://www.scielo.br/pdf/tce/v26n3/0104-0707-tce-26-03-e1090016.pdf 
Research, Society and Development, v. 10, n. 9, e2810917803, 2021

(CC BY 4.0) | ISSN 2525-3409 | DOI: http://dx.doi.org/10.33448/rsd-v10i9.17803

Souza, G. R. M., et al. (2017) Atuação dos enfermeiros da estratégia saúde da família na atenção oncológica. Esc. Anna Nery vol.21 no.4 https://doi.org/10.1590/2177-9465-ean-2016-0380

Souza, M. T., et al. 2010 Revisão integrativa: o que é e como fazer 1. einstein. 8(1 Pt 1):102-6. https://journal.einstein.br/pt-br/article/revisao-integrativa-o-quee-e-como-fazer/

Valentim, L. V., et al. (2020). Percepção dos profissionais de enfermagem quanto ao trabalho em equipe. Rev baiana enferm; 34:e37510. https://pesquisa.bvsalud.org/portal/resource/pt/biblio-1137042

Wakiuchi, J., et al. (2016). Atenção a pacientes oncológicos na Estratégia Saúde da Família: olhar do usuário. Rev. Gaúcha Enferm. https://doi.org/10.1590/19831447.2016.01.54088.

Wakiuchi, J., et al. (2016). Atuação da estratégia saúde da família na perspectiva de usuários com câncer. Rev. Eletr. Enf. http://dx.doi.org/10.5216/ree.v18.38612. 